\title{
O NÚMERO $\pi$ E A QUADRATURA DO CÍRCULO
}

\section{THE $\pi$ NUMBER AND THE CIRCLE QUADRATURE}

Juliano Ferreira de Lima, Amanda Santos Silva, Fernando Pereira de Souza

Universidade Federal de Mato Grosso do Sul - UFMS, Curso de Licenciatura em Matemática, Três Lagoas, MS

E-mail: juliano.ferreira.info2@gmail.com

RESUMO - Há muito tempo, cerca de 4000 a.C, já existia o número $\pi$, porém, não como conhecemos hoje. Vale ressaltar que o primeiro matemático a utilizar o número "pi" como sendo a letra grega $\pi$ foi o matemático francês Leonhard Euler (1707-1783). Tão antigo quanto o valor de $\pi$ é o celebre problema grego da quadratura do círculo, ou seja, utilizando régua e compasso construir um quadrado de área igual à de um círculo dado. Para resolver essa questão, utilizaremos a ideia de números transcendentes e principalmente o fato de $\pi$ ser um número transcendente, ou seja, ele não pode ser raiz de uma equação algébrica com coeficientes inteiros, fato que foi provado por o matemático alemão Ferdinand von Lindemann em 1882, desta forma, mostraremos que é impossível resolver tal problema.

Palavras-chave: Problema Grego; Número $\pi$; Números Transcendentes; Geometria Plana; Áreas.

Recebido em: 19/08/2015

Revisado em: 31/08/2015

Aprovado em: 14/09/2015
ABSTRACT - Long ago, around $4000 \mathrm{BC}$, there was already the number $\pi$, but not as we know it today. It is noteworthy that the first mathematician to use the number "pi" as the Greek letter $\pi$ was the French mathematician Leonhard Euler (1707-1783). As old as the $\pi$ value is the celebrated Greek problem of squaring the circle, ie using ruler and compass to construct a square equal to the area of a given circle. To address this issue, we will use the idea of transcendental numbers and especially the fact that $\pi$ is a transcendental number, meaning it can not be the root of an algebraic equation with integer coefficients, a fact proved by the German mathematician Ferdinand von Lindemann in 1882 thus show that it is impossible to solve such a problem.

Keywords: Greek Problem; Number $\pi$; Numbers Transcendental; Plane Geometry; Areas. 


\section{INTRODUÇÃO}

Neste presente trabalho iremos apresentar um dos três problemas clássicos grego "a quadratura do círculo", ou seja, construir utilizando régua e compasso, um quadrado cuja área fosse igual à de um circulo dado. Esses problemas clássicos desempenharam papel significativo na matemática, em particular, no estudo da Geometria.

Todos os problemas deveriam ser resolvidos apenas utilizando régua $\mathrm{e}$ compasso, pois, esses eram os únicos instrumentos utilizados por Euclides de Alexandria (pai da geometria).

O trabalho está organizado da seguinte forma, no primeiro momento iremos apresentar o que é o número $\pi$, em seguida, abordaremos de forma simples o que são os números transcendentes e por fim o problema grego.

\section{METODOLOGIA}

O trabalho é resultado de uma pesquisa teórica que foi abordada no Trabalho de Conclusão de Curso (TCC) no começo do primeiro semestre de 2015 cujo tema é: "Trigonometria com aplicações para o ensino médio", desenvolvido através de discussões do tema com o orientador.

0 estudo e as atividades desenvolvidas foram avaliados através da apresentação de seminários de discussão e rodas de conversas.

\section{RESULTADOS}

\section{O que é o número $\pi$ ?}

Segundo o Professor Elon no livro "Explorando a matemática", a maneira mais rápida de responder a essa pergunta é dizer que $\pi$ é o valor da área de um círculo de diâmetro 1 , ou seja, se um círculo tem raio igual a $\frac{1}{2} \mathrm{~cm}$ então sua área é dada por $\pi$ $\mathrm{cm}^{2}$. Também podemos dizer que se $C$ é uma circunferência, então $\pi=\frac{p}{d}$, onde $p=$ perímetro de $C$ e $d=$ diâmetro de $C$, já que o quociente entre comprimento e o diâmetro de qualquer circunferência resulta em um mesmo valor, fato este notado a cerca de 4000 anos.

Antigamente não se havia um padrão de notação para a razão $\frac{p}{d}$ mencionada, sendo que o grande matemático Euler costumava usar $\pi$ ou $c$ para denotar essa constante. Posteriormente ele passou a usar constantemente a letra grega $\pi$ para denotar essa constante, e a partir daí foi seguido pelos outros matemáticos.

Atualmente sabe-se que o seu valor tem uma aproximação dada por $3,141592653 \ldots$... sendo que ja era de conhecimento dos babilônios que:

$$
\frac{25}{8} \leq \pi \leq \frac{22}{7} \Rightarrow 3,125<\pi<3,142 .
$$


Desde Arquimedes que obteve o valor $\pi=3,1416$, alguns matemáticos tem se ocupado da desgastante tarefa de calcular o $\pi$ com uma precisão cada vez maior. Um destes matemáticos foi o Inglês Willian Shanks que calculou $\pi$ com 707 algarismos decimais exatos em 1873. Posteriormente com o auxílio de máquinas manuais foi calculada uma aproximação de $\pi$ com 808 algarismos. Já com o auxílio de computadores foi obtida em 1967 na França, uma aproximação de $\pi$ com 800.000 algarismos e em 1984 nos estados unidos uma aproximação com 10.013.395 algarismos decimais exatos.

Neste ponto cabe uma pergunta: Quantos algarismos decimais são necessários para ter o valor exato de $\pi$ ?

Obviamente a resposta para essa pergunta é que não é possível determinar uma quantidade de algarismos decimais de forma a determinar o valor exato de $\pi$, já que se sabe que o mesmo é um número irracional (provado pelo matemático suíço Johann Heinrich Lambert em 1761) e, portanto, nenhuma fração decimal finita ou periódica pode representar o seu valor exato.

Em outras palavras, não importa quantos algarismos decimais tomemos, nunca obteremos uma periodicidade da dízima e muito menos o valor exato de $\pi$, somente encontraremos aproximações.
A resposta da pergunta anterior sugere outra pergunta: Se não é possível determinar exatamente o valor de $\pi$, então porque calcular $\pi$ com centenas ou milhares de algarismo decimais?

Uma possível resposta é a motivação parte do desejo de "superar" a aproximação feita por outro, motivo pelo qual existe, por exemplo, o livro dos recordes do Guinness.

Outra resposta é o de testar o bom funcionamento de computadores, fazendo com que calculem o valor de $\pi$ e comparando com os valores já conhecidos.

Euler acreditava na irracionalidade $\pi$, assim, ele apontou que $\pi$ seria transcendente.

Def. (MARQUES, 2013) Um número é dito algébrico se ele for raiz de um polinômio, não nulo, com coeficientes inteiro, caso contrario, ele é dito transcendente.

Em outras palavras, se $p(x)$ não é solução de:

$$
\begin{gathered}
p(x)=a_{0}+a_{1} x+a_{2} x^{2}+\cdots+a_{n} x^{n}, a_{i} \\
\qquad \in \mathbb{Z}, i \in \mathbb{N}, \\
\text { então } p(x) \text { é transcendente. }
\end{gathered}
$$

\section{A quadradura do círculo}

Um antigo problema grego conhecido como quadratura do círculo é: Construir com auxílio de régua e compasso, um quadrado cuja área fosse igual à de um círculo dado. 
Iremos fazer agora algumas compasso, a partir de um segmento dado, considerações a respeito deste problema, tomado como unidade, outro segmento de para isto diremos "construir um número $x$ ", comprimento igual a $x$ ". para significar "construir utilizando régua e

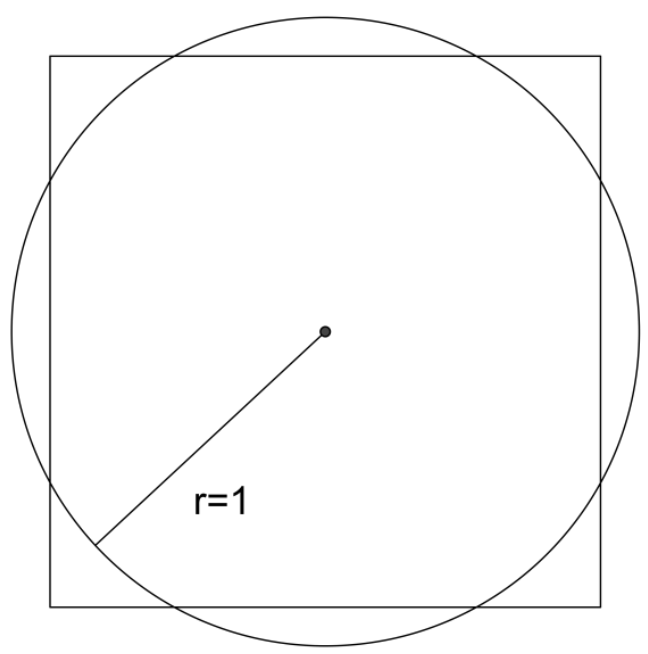

Figura1. Quadratura do círculo.

Tomando o raio do círculo como unidade de comprimento, o problema de quadratura do círculo equivale a pedir que se construa, com régua e compasso, um segmento de comprimento igual a $\sqrt{\pi}$, pois, a área do circulo será dada por $A_{c}=\pi$ e como queremos que a área do quadrado seja igual a do círculo, então:

$$
A_{q}=l . l \Rightarrow \pi=l^{2} \Rightarrow l=\sqrt{\pi} .
$$

Assim, o problema da quadratura do círculo se torna o problema de construir o número $\sqrt{\pi}$ equivalente ao lado do quadrado.

Segundo Barbosa (2006), as construções geométricas feitas com régua e compasso são basicamente retas e circunferências, ou seja, são obtidas a partir das seguintes operações básicas:

- Traçar retas por dois pontos dados;

- Traçar uma circunferência com centro e raio dado.

Considerando o plano cartesiano temos:

- $y=a x+b$ (retas);

- $\left(x-a_{0}\right)^{2}+\left(y-b_{0}\right)^{2}=r^{2}$

(circunferências).

Assim, um número que se pode construir é sempre obtido como solução de um sistema de 2 equações a 2 incógnitas cujos graus são $<2$. 


$$
\left\{\begin{array}{l}
y=a x+b, \\
\left(x-a_{0}\right)^{2}+\left(y-b_{0}\right)^{2}=r^{2} .
\end{array}\right.
$$

Logo, se um número real $x$ pode ser construído, então $x$ é obtido de um número finito de operações de adição, subtração, multiplicação, divisão e extração de raiz quadrada, realizadas a partir de números inteiros.

Portando, todo número que pode ser construído com régua e compasso é raiz de uma equação da forma $\alpha x^{2}+\beta x+\gamma=$ $0, \alpha, \beta$ e $\gamma \in \mathbb{Z}$, ou seja, todo número construído com régua e compasso é algébrico.

Suponhamos que $x=\sqrt{\pi}$, seja algébrico, logo é raiz de um polinômios com coeficientes inteiros, em particular, de grau 2:

$$
\begin{gathered}
\alpha(\sqrt{\pi})^{2}+\beta \sqrt{\pi}+\gamma=0, \\
\alpha \pi+\beta \sqrt{\pi}+\gamma=0 \Rightarrow \beta \sqrt{\pi}=-\alpha \pi-\gamma, \\
\text { elevando ambos os lados ao }
\end{gathered}
$$

quadrado:

$$
(\beta \sqrt{\pi})^{2}=(-\alpha \pi-\gamma)^{2},
$$

desenvolvendo o quadrado, podemos reescrever a equação anterior como:

$$
\alpha^{2} \pi^{2}+\pi\left(2 \alpha \gamma-\beta^{2}\right)+\gamma^{2}=0 .
$$

Desta forma, podemos observar que $\pi$ é solução da seguinte equação do 20 grau:

$$
\alpha^{2} y^{2}+\left(2 \alpha \gamma-\beta^{2}\right) y+\gamma^{2}=0 .
$$

É um absurdo, pois, é uma equação do $2^{\circ}$ grau com coeficientes inteiros tornando $\pi$ um número algébrico.
Portanto, $\sqrt{\pi}$ é transcendente, então não satisfaz o sistema da quadradura do círculo tornando o problema impossível.

\section{DISCUSSÃO}

O problema da quadradura do círculo exerce um impacto importante na motivação para atrair a atenção dos alunos principiantes, pois vale ressaltar que o principal foco é fazer com que os alunos pensem se realmente é ou não possível construir com régua e compasso um círculo com área igual de um quadrado.

Muitos problemas atraentes de matemática exploram relações entre sistemas de equações, expressas em linguagem coloquial. Parte de sua atração vem justamente do fato de que podem ser formulados e, muitas vezes, resolvidos sem recorrer a fórmulas ou a técnicas complicadas.

\section{CONCLUSÃO}

Neste trabalho abordamos um pouco da história do número $\pi$, o que são números transcendentes e por fim a quadradura do círculo, como vimos, é impossível fazer a quadradura utilizando apenas régua $\mathrm{e}$ compasso. Embora pareça simples, é através dele que podemos demonstrar resultados possivelmente inesperados e resolver vários exercícios curiosos, tornando assim o ensino mais prazeroso. 
Acreditamos que nosso objetivo foi atingido e como resultado se obteve um texto claro, bem estruturado e acessível a diversos estudantes. Este trabalho é de extrema importância, pois permite um aprofundamento sobre os números transcendentes.

\section{REFERÊNCIAS}

BARBOSA, J. L. W. Geometria Euclidiana Plana. 10. ed. Rio de Janeiro: Markgraph, 2006.

MARQUES, D. Teoria do Números

Transcendentes. 1. ed. Rio de Janeiro: SBM, 2013. 